University at Buffalo School of Law

Digital Commons @ University at Buffalo School of Law

$12-1-1996$

\title{
Avocats et divorce aux Etats-Unis: la transformation des pratiques professionnelles
}

Lynn M. Mather

University at Buffalo School of Law

Craig A. McEwen

Bowdoin College

Richard J. Maiman

Follow this and additional works at: https://digitalcommons.law.buffalo.edu/journal_articles

Part of the Family Law Commons, and the Legal Profession Commons

\section{Recommended Citation}

Lynn Mather, Craig A. McEwen \& Richard J. Maiman, Avocats et divorce aux Etats-Unis: la transformation des pratiques professionnelles, Droit et Société, Dec. 1996, at 341.

\section{C) ${ }_{\text {COPYRIGHT }}^{\text {N }}$}

This Article is brought to you for free and open access by the Faculty Scholarship at Digital Commons @ University at Buffalo School of Law. It has been accepted for inclusion in Journal Articles by an authorized administrator of Digital Commons @ University at Buffalo School of Law. For more information, please contact lawscholar@buffalo.edu. 


\section{Avocats et divorce aux États-Unis : \\ la transformation des \\ pratiques professionnelles *}

\section{Lynn Mather **, Richard J. Maiman, Craig A. McEwen}

\section{Résumé}

Les transformations sociales et les évolutions juridiques qui sont intervenues aux États-Unis depuis les années 60 ont eu de multiples effets sur le travail des avocats en matière de divorce. Le présent article analyse ces transformations en s'appuyant sur des entretiens avec des avocats et sur l'analyse de l'activité des tribunaux dans les États du Maine et du New Hampshire. Il souligne notamment l'importance que revêt l'accroissement du nombre des divorces parmi les couples ayant des ressources moyennes ou faibles. Il décrit aussi la féminisation rapide du barreau, une tendance qui se trouve particulièrement accentuée en ce qui concerne les spécialistes du divorce. Sont également évoquées les règles nouvelles qui s'appliquent à la garde des enfants et à la division du patrimoine familial. L'analyse faite suggère que le rôle des avocats en matière de divorce s'est fortement différencié en fonction des catégories sociales qui constituent leur clientèle et du degré de leur spécialisation; elle met en évidence le développement d'une approche féministe du travail de l'avocat du divorce, à l'initiative de quelques avocates, en même temps qu'elle montre que les changements légaux et l'introduction de la médiation ont réduit le conflit judiciaire dans ce domaine.

Avocats - Divorce - Féminisme - Médiation - Spécialisation légale.

\section{Summary}

Changes in Divorce Law and Legal Practice in the United States

Social and legal changes in the United States since the 1960's have affected the practice of divorce law in a variety of ways. This article analyzes these developments by drawing on interview data and court records from the states of Maine and New Hampshire. Prominent among the changes examined are the increased incidence of divorce among middle and lower income couples; the rapid rise in the number of women attorneys, a trend particularly notable among divorce specialists; new rules governing child custody and division of marital property; an increased

\section{Les auteurs}

Lynn Mather

Professor of Government at Dartmouth College, in Hanover, New Hampshire.

Richard J. Maiman

Professor of Political Science and Associate Provost for Graduate Studies and Research at the University of Southern Maine, in Portland, Maine.

\section{Craig A. McEwen}

The Daniel B. Fayerweather Professor of Sociology at Bowdoin College, in Brunswick, Maine.

Professors Mather, Maiman and McEwen are completing a book about legal professionalism and have jointly published several recent articles about divorce lawyers, including :

- « Gender and Specialization in the Practice of Divorce Law ", Maine Law Review, vol. 44, n 1, 1992 ;

- "Lawyers, Mediation, and the Management of Divorce Practice ", Law and Society Review, vol. $28, n^{*} 1,1994$;

- " "The Passenger Decides on the Destination and I Decide on the Route" : Are Divorce Lawyers "Expensive Cab Drivers" ?", International Journal of Law and the Family, vol. 9, n' 3, 1995.

" Traduit par Benoit Bastard.

** Dartmouth College,

Department of Government, 6108 Silsby Hall, Hanover, Vew Hampshire 03755, USA. 
L. Mather, R.J. Maiman,

C.A. McEwen

Avocats et divorce

aux États-Unis :

la transformation des

pratiques professionnelles

1. Pour un exposé général sur les professions légales voir, par exemple, R. ABEL, American

Lawyers, New York, Oxford University Press, 1989 et

R. NELSON, D. TRUBEK et

R. SOLOMON, Lawyer's Ideals/

Lawyer's Practices : Transforma-

tion in the American Legal

Profession, Ithaca, Cornell

University Press, 1992.

Beaucoup de la recherche et des écrits sur la transformation de la pratique du droit aux ÉtatsUnis a porté sur le développement des grandes firmes d'avocats ou le fonctionnement des barreaux et non sur des domaines particuliers du droit. voir, par exemple, M. GALANTER et T. PALAY, Toumament of Lawyers : The Transformation of the Big Law Firm, Chicago, University of Chicago Press, 1991 ; R. NELSON, Partners with Power: The Social

Transformation of Large Law Firms, Berkeley, University of California Press, 1988 et

T. HALLIDAY, Beyond Monopoly: Lawyers, State Crises, and Professional Empowerment, Chicago, University of Chicago Press, 1987.

2. Ce travail présente les résultats d'une recherche financée par les subventions SES-891025, SES-8910649 et SES-8911653 de la Law and Social Sciences Division de la National Science Foundation ainsi que par le Rockefeller Center for the Social Sciences au Dartmouth College. Les points de vue présentés sont ceux des auteurs et n'engagent pas nécessairement la National Science Foundation. use of mediation in divorce cases. The analysis shows, for example, how the roles and work of divorce lawyers have become differentiated according to the class of clientele and degree of legal specialization, how some women lawyers have brought a new style of feminist advocacy to divorce, and how legal changes and the introduction of mediation have reduced adversarial legal conflict in divorce.

Divorce - Feminism - Lawyers - Legal specialization - Mediation.

Les transformations d'ensemble de la profession d'avocat ainsi que les changements plus spécifiques au domaine du divorce ont eu un impact sur l'activité des avocats dans ce domaine ${ }^{1}$. De multiples évolutions se sont produites depuis trente ans dans la pratique du divorce aux États-Unis. Cet article ${ }^{2}$ examine plus particulièrement quatre types de changement : 1) l'accroissement du nombre des divorces, en particulier dans les catégories sociales les plus favorisées, qui s'est traduit non seulement par un élargissement de ce "marché ", mais aussi par un accroissement des revenus potentiels que les avocats peuvent espérer en retirer ; 2) l'émergence d'un courant féministe au sein du barreau à la faveur de la féminisation de la profession et la revendication de droits nouveaux pour les femmes en matière de divorce; 3 ) l'introduction de changements législatifs de grande envergure qui ont conduit à la quasidisparition de la notion de faute en matière de divorce et ont amené une redéfinition des modalités de répartition des biens et l'instauration, en ce qui concerne les enfants, de " présomptions en matière de garde » ainsi que de nouveaux modes de calcul des pensions alimentaires ; 4) enfin, la transformation des institutions qui prennent en charge le traitement du divorce, à la faveur de l'émergence de la médiation et du développement de préoccupations accrues au sujet des problèmes qui se posent aux enfants dans les situations de divorce.

Toutes ces évolutions posent question aux avocats et constituent des opportunités pour eux. Considérées dans leur ensemble, elles aident à mieux comprendre les tendances actuelles de la pratique du divorce.

Pour les analyser, nous nous sommes appuyés sur des entretiens réalisés auprès d'avocats ainsi que sur l'étude des dossiers des juridictions du divorce dans le Maine et le New Hampshire, deux États de petite taille, situés au nord-est des États-Unis et ayant une population en majorité rurale. Les entretiens ont été conduits auprès de 163 avocats (dont un tiers de femmes) par les trois auteurs, en 1990-1991. Bien que les données recueillies au moyen de ces entretiens ne présentent pas un caractère longitudinal, elles constituent néanmoins une source pour analyser les changements de la pratique du divorce, notamment parce que les 
avocats tiennent toutes sortes de propos au sujet des transformations de leur activité à travers le temps. Nous avons également dépouillé les dossiers constitués par les juridictions pour un large échantillon de divorces, dans les mêmes circonscriptions (counties) où nous avons réalisé les entretiens. 7000 affaires ont été étudiées, correspondant à une période de 9 ans (de 1979 à 1988).

\section{Les transformations des modalités du divorce et de la représentation des conjoints}

Aux États-Unis, les taux de divorce ont augmenté régulièrement des années 60 aux années 80. Par exemple, le taux de divorce, pour 1000 femmes mariées âgées de 15 ans et plus, est monté de 9,2 en 1960 à 22,8 en 1979 ; après quoi, il est resté stable pour se fixer ensuite à 21,2 en 1986. L'accroissement des taux n'est pas tout: dans le même temps, le volume des divorces est passé de 393000 en 1960 à 1200000 en 1980, soit une augmentation de près de $280 \%{ }^{3}$. Un tel chiffre marque encore mieux l'accroissement sans précédent de la divortialité.

L'augmentation du nombre des divorces a eu un impact direct sur la pratique des avocats dans ce domaine : pour chaque divorce, en effet, il y a virtuellement de l'emploi pour deux avocats. Entre 1960 et 1980 , le nombre des avocats a pratiquement doublé aux États-Unis ${ }^{4}$. Cet accroissement, allant de pair avec celui du volume des divorces et avec la tendance à la spécialisation du travail des avocats, a poussé beaucoup d'entre eux à se spécialiser dans ce domaine. Auparavant, seule une minorité d'avocats étaient des spécialistes du divorce et des affaires matrimoniales. Dans son étude pionnière sur les avocats de la famille à New York à la fin des années 50, O'Gorman indique que très peu de ceux qui traitent des affaires de divorce sont "spécialisés " 5 . En décrivant les avocats à Chicago, à peu près à la même époque, Jérôme Carlin note que « à Chicago, on trouve environ 12 spécialistes du divorce " 6 . Bien qu'aucune comparaison systématique ne soit possible, il est sûr que la spécialisation s'est accrue au cours des trente-cinq dernières années dans le domaine du droit de la famille ${ }^{7}$. Cela dit, ce n'est pas seulement l'augmentation du nombre des ruptures familiales qui a changé la pratique du divorce pour les avocats, mais c'est également la transformation qualitative de la population des divorçants.

Avant les années 60, la probabilité du recours au divorce était inversement proportionnelle au revenu ${ }^{8}$. Autrement dit, à une époque où la fréquence du phénomène était faible dans l'ensemble de la population, le divorce était spécialement peu répandu parmi les personnes disposant de revenus assez hauts. Durant les années 60 , on a assisté à une sorte de "démocratisation " du divorce, dans
3. NATIONAL CENTER FOR HEALTH STATISTICS, Vital Statistics of the United States. 1986. v. III. Marriage and Divorce, Washington, U.S. Government Printing Office, 1990, tableau 2-1.

4. R. ABEL, op. cit., p. 280.

5. H. O'GORMAN, Lawyers and Matrimonial Cases : A Study of Informal Pressures in Private Professional Politics, Glencue, Free Press, 1963, p. 65-80.

6. J. CARLIN, Lawyers on Their Own, New Brunswick, Rutgers University Press, 1962, p. 95.

7. Par exemple, on comptait au moins 12 spécialistes du droit de la famille en 1990 à Portland, Maine, autrement dit dans une zone urbaine représentant environ le vingtième de la population de Chicago en 1960.

8. R. UDRY, "Research Notes: Marital Instability by Race and Income Based on 1960 Census Dala ", American Journal of Sociology, vol. 72,1967, p. 673. 
L. Mather, R.J. Maiman,

C.A. McEwen

Avocats et divorce

aux États-Unis :

la transformation des

pratiques professionnelles

9. P. GIICK, "A Demographic Look at American Families", Journal of Marriage and the Family, vol. 37, 1975, p. 19.

10. U.S. BUREAU OF THE CENSLS, U.S. Census of Population: 1980. Subject Reports. Marital Characteristics. Final Report PC80(2)-4C, Washington, U.S. Government Printing Office, 1984, tableau 6.

11. U.S. BUREAU OF THE CENSL'S, U.S. Census of Population: 1960. Subject Reports. Marital Status. Final Report PC (2)-4E, Washington, U.S. Government Printing Office, 1966, tableau 6.

12. E. Lalmann et J. Heinz, "Specialization and Prestige in the Legal Profession: The Structure of Deference ", American Bar Foundation Research Journal, 1977, p. 167. le sens où l'écart s'est comblé entre le taux de divorce des catégories à revenus faibles et celui des catégories à revenus élevés ou encore entre les personnes disposant de capitaux scolaires et les autres 9. En 1979, par exemple, plus de 2 millions d'hommes divorcés disposaient d'un revenu annuel supérieur à 25000 dollars ${ }^{10}$. On peut rapprocher ce chiffre de celui de 1960: 450000 hommes divorcés disposant de 10000 dollars ou plus ${ }^{11}$. L'accroissement de demandes en direction des avocats provenant de divorçants disposant de revenus moyens ou élevés a permis à bon nombre d'avocats non seulement de se spécialiser dans ce domaine du divorce, mais aussi de pratiquer exclusivement pour cette clientèle plus aisée.

L'augmentation du nombre des ruptures familiales et la transformation du statut socio-économique des divorçants vont de pair avec d'autres évolutions dans le domaine du divorce. L'avènement du "no-fault divorce " a notablement réduit la part du " sale travail " dans l'activité des avocats. La moindre stigmatisation qui s'attache au traitement des affaires de divorce ajoutée au fait qu'un nombre croissant de divorçants se trouvaient en position de dépenser davantage en services juridiques ont permis l'émergence d'une élite des avocats spécialisés dans ce domaine au plan national comme au plan local. L'un des projets principaux de ces avocats a été d'obtenir la reconnaissance du prestige de cee type de travail, une entreprise particulièrement difficile compte tenu de son statut très bas au départ. Dans l'étude de Laumann et Heinz sur les avocats de Chicago, au milieu des années 70), le divorce n'occupait que la $29^{\circ}$ position en termes de prestige parmi les 30 qui étaient soumises à l'appréciation d'un échantillon d'avocats en activité $^{12}$. La création de la section de droit de la famille (Family Law Section) au sein de l'ordre des avocats (American Bar Association), en 1957, et la création de la très prestigieuse Academy of Matrimonial Lawyers, en 1962, ont fourni les premières bases institutionnelles qui ont permis de construire l'excellence du barreau en matière de divorce. La section de droit de la famille a connu son recrutement maximum dans le prolongement de l'adoption du nofault divorce, au beau milieu de la vague d'accroissement du nombre des divorces. Dans la période 1976-1987, elle est en effet passée de 5000 membres à 1600() .

Le développement de la spécialisation des avocats en matière de divorce et de droit de la famille au cours des deux dernières décennies s'est accompagné de la segmentation de différents types de pratique, en fonction soit de la catégorie sociale et des ressources des clients, soit de la distinction entre les avocats spécialisés et le grand nombre des avocats qui prennent des divorces dans le cadre d'une pratique généraliste. Ces distinctions se recouvrent, dans la mesure où les spécialistes représentent les clients ayant les plus hauts revenus. Dans notre échantillon d'avocats, les spécialis- 
tes du divorce (ceux qui réalisent les trois quarts de leur activiti dans ce domainel sont presque quatre fois plus nombreux que les avocats ayant une activité moindre en matiere de divorce à indiquer que leur clientèle est constituce par les catégories sociales les plus aisées. La spécialisation des avocats et la constitution de clientèles de divorçants appartenant aux catégories les plus aisées ont certainement participé à la transformation du processus de divorce et à la redéfinition du rôle des avocats.

Les avocats - dont le nombre reste relativement petit - qui ont une clientèle de divorçants aisés ont des études confortables avec des cuvres d'art au mur. Par contraste, les avocats, beaucoup plus nombreux et peu spécialisés, qui ont pour clients les classes moyennes et populaires ont des bureaux plus modestes, souvent tristes, voire vétustes.

Dans le Maine et le New Hampshire, en 1990, les $20 \%$ d'avocats qui indiquent que leurs clients appartiennent aux catégories sociales les plus aisées (upper middle class) demandaient 125 dollars par heure en moyenne, alors que les $40 \%$ d'avocats dont la clientèle provient des classes moyennes et populaires (working class and middle class clients) demandaient en moyenne 95 dollars. Les honoraires demandés au départ ( retainer») par les avocats ayant les clients aux revenus les plus élevés étaient en moyenne de 1617 dollars, le triple de ceux demandés par les avocats ayant les clients les moins fortunés (516 dollars en moyenne). Les différences importantes de ces montants reflètent davantage que la simple différence des taux applicables à l'heure de travail. Elles correspondent plutôt aux représentations très contrastées que se font les avocats du nombre d'heures qu'ils estiment nécessaire de consacrer à un dossier de divorce. Nous avons demandé aux avocats d'estimer quelle proportion des affaires de divorce de leur cabinet nécessite dix heures de travail ou moins et quelle proportion nécessite vingt heures ou plus. Parmi les avocats qui ont la clientèle la plus aisée (upper and upper middle class), très peu nous ont dit avoir des affaires exigeant dix heures de travail ou moins, alors que plus de la moitié des avocats ayant des clients de classe populaire nous ont répondu que la plupart des affaires de divorce de leur cabinet se traitent dans de tels délais. À l'inverse, pour $73 \%$ des avocats du premier groupe, c'est plus de la moitié des affaires qui demande 20 heures de travail ou davantage, alors que cette proportion n'est que de $15 \%$ chez les avocats qui ont les clients aux revenus les plus modestes.

Les avocats dont la clientèle dispose de revenus moyens ou faibles ont tendance à travailler en gardant à l'esprit la limite de ce que leurs clients peuvent payer pour leur travail et réduisent par conséquent aussi bien leur taux horaire que le nombre d'heures qu'ils passent sur chaque dossier. Les avocats qui ont les clients aisés ont des marges beaucoup plus importantes; ils prennent plus 
L. Mather, R.J. Maiman,

C.A. McEwen

Avocats et divorce

aux États-Unis :

la transformation des

pratiques professionnelles cher et passent beaucoup plus d'heures sur chaque dossier. L'idéologie professionnelle qui a cours au sujet du divorce justifie cette différence en faisant état du fait que les besoins en matière juridique ne sont pas comparables suivant que l'on a affaire à des couples qui ont des biens ou à d'autres qui n'en ont pas. Autrement dit, les clients qui ont des revenus élevés sont aussi ceux qui possèdent davantage de biens et ceux dont le divorce pose des problèmes juridiques plus complexes.

"Dès que vous avez affaire à une classe sociale différente, les affaires qui font problème ne sont plus celles dans lesquelles il y a des difficultés à propos des enfants. Les divorces compliqués et qui demandent beaucoup de temps sont ceux dans lesquels vous avez à décider comment diviser les droits à la retraite ou encore dans lesquels vous avez des biens qui n'appartiennent pas à la communauté et qu'il faut estimer. Ces divorces-là, avec des problèmes au sujet des biens, sont ceux qui me prennent le plus de temps. » (Un avocat)

Dans de telles situations, le temps de travail des avocats - et par conséquent le coût et la facture - augmente en même temps que la capacité du client à faire face à de telles dépenses. À l'inverse, les personnes ayant des revenus faibles demandent moins de travail à l'avocat et laissent moins d'argent chez lui.

« Je parle de la clientèle que je représente, la classe moyenne ou les pauvres. Ils n'ont ni biens, ni revenus - rien qui puisse valoir qu'on dépense énormément d'argent. » (Un avocat)

Dans la transformation du marché professionnel du divorce et des conceptions qui prévalent au plan juridique et judiciaire, le rôle et le travail des avocats se sont donc différenciés d'une manière très nette. En particulier, l'élite des avocats de la famille exerçant leur activité pour une clientèle aisée pratique selon des modalités qui incluent des honoraires de départ substantiels, des taux horaires élevés et un nombre d'heures de travail important pour traiter chaque cas. Ces avocats, à la différence des autres, ne sont pas confrontés au problème que posent les relations avec un client qui a du mal à régler leurs honoraires et ils n'ont pas non plus à faire des choix difficiles en réduisant leurs prestations de façon à parvenir au divorce avec le moins possible d'heures de travail sur la facture. En pratique, ces spécialistes du divorce pour clientèle aisée empruntent beaucoup de la manière de conduire la procédure aux avocats du domaine civil et font un très grand usage d'interrogatoires, de dépositions et de requêtes. Les données recueillies dans les dossiers des juridictions confirment sur ce point les propos tenus par les avocats. En recourant à davantage de requêtes et d'audiences, les membres de l'élite professionnelle des avocats du divorce font en sorte que leur activité ressemble à la " vraie » pratique du droit. En accroissant le formalisme, ils aug- 

leur savoir-faire.

En même temps, les différences dans les ressources mises en jeu et dans les modalités de travail des uns et des autres divisent le barreau et poussent les avocats généralistes qui ont les clients aux revenus faibles à entrer en conflit avec leurs confrères de l'élite lorsqu'ils sont face à face dans certaines affaires. Une préoccupation constante de nombre d'avocats qui ont les clients les moins favorisés est de savoir comment limiter les actes de procédure de manière à diminuer le temps passé sur chaque dossier. C'est pourquoi ces avocats dénoncent la tendance à la spécialisation en matière de divorce, qu'ils rendent responsable de l'accroissement considérable du formalisme, des difficultés et des dépenses dans ce type d'affaires.

" Le travail en matière de divorce devient très difficile maintenant, parce que s'est développé dans le New Hampshire un corps d'avocats qui se spécialisent dans le divorce. Cela rend très difficile le règlement de pas mal d'affaires qui, de mon point de vue, sont des divorces qui se poursuivent pour trois fois rien et qu'on aurait déjà pu régler normalement il y a cinq ou dix ans. Dans le temps, on aurait eu un échange de lettres entre attorneys, des coups de téléphone, et on se serait dit : voici ce que mon client possède. Aujourd'hui, cela se fait à coup d'interrogatoires. »

Les interrogatoires consistent en un ensemble de questions formelles qui est soumis à la partie adverse pour obtenir des informations sur sa situation. La partie est obligée de fournir des réponses dans des délais assez brefs. Les spécialistes ont introduit cette formule de l'interrogatoire dans les procédures de divorce à la consternation des avocats généralistes.

Bon nombre de ces derniers, habitués qu'ils sont à traiter toutes sortes d'affaires d'une manière informelle et " confraternelle ", pensent que les divorces doivent être réglés de manière rapide et sans exagérer les problèmes de détail. Pour eux, ces affaires-là ne diffèrent guère des contrats ou des affaires immobilières qu'ils gèrent tous les jours. Un de ces avocats se plaint dans les termes suivants d'un de ces spécialistes qu'il juge déraisonnable :

"C'en est un qui fait une montagne avec rien. Il y en a beaucoup qui sont comme ça dans les divorces. Je ne comprends pas : à quoi ça sert de passer cinq heures à négocier un bien d'une valeur de 1200 dollars, si ça doit coûter plus de 1200 dollars aux deux clients réunis? C'est absurde.»

Ces commentaires, comme beaucoup d'autres, reflètent des préoccupations qui renvoient autant à des différences sociales entre les clients qu'à des différences dans le degré de spécialisation des professionnels. Les généralistes qui travaillent pour les clients les plus pauvres doivent être particulièrement prudents au 
L. Mather, R.J. Maiman, C.A. McEwen Avocats et divorce aux États-Unis : la transformation des pratiques professionnelles
13. R. ABEL, op. cit., p. 286. niveau des coûts de la procédure et des heures facturées dès lors qu'ils savent qu'ils ne pourront pas se faire payer au-delà d'une dizaine d'heures de travail environ.

À l'inverse, les spécialistes du divorce se voient comme une catégorie à part, très différente de la masse des avocats qui prennent des divorces à l'occasion. Ils considèrent qu'ils possèdent une vue large sur tout un ensemble de problèmes : la complexité de la division des biens, les risques et les difficultés de la conduite de la procédure de divorce, les aspects psychologiques de la séparation et l'importance que revêt le divorce pour les parties qui s'affrontent. Les membres de cette élite défendent, par exemple, l'usage qu'ils font des interrogatoires par la nécessité d'obtenir des informations complètes sur les valeurs détenues par les parties. Certains insistent aussi sur le fait que les interrogatoires donnent aux avocats une protection contre les attaques pour faute professionnelle. Les avocats spécialisés se connaissent entre eux et préfèrent en outre avoir l'un des leurs en face d'eux. Ils critiquent les généralistes qui, selon eux, ne savent pas distinguer ce qui importe pour les divorçants et ne montrent aucune sensibilité pour la spécificité du travail dans ces affaires de divorce:

" Je n'aime pas les avoir en face. Beaucoup de ces gens ne travaillent pas comme il le faudrait dans un divorce. Ils s'opposent à une demande légitime de l'autre avocat, parce que cela demanderait du temps. Il va y avoir sans arrêt des problèmes dans l'instruction de l'affaire, avec ce genre d'avocats. Ils ne vont pas répondre à une demande d'information tout à fait légitime et raisonnable, si bien qu'il va falloir introduire une requête, les amener au tribunal et faire dire au juge qu'ils doivent répondre, ou encore trouver un moyen détourné d'avoir cette information. J'aime mieux travailler avec lles spécialistes du divorce] parce que je sais où je vais. » (Un avocat spécialisé dans le droit de la famille)

Alors que les généralistes pensent que les spécialistes sont trop formalistes et trop attachés au détail, les spécialistes, eux, voient les généralistes comme insensibles aux problèmes des clients et trop désinvoltes dans leur façon de traiter ces affaires.

\section{Le féminisme et les droits des femmes dans la législation du divorce}

Le mouvement féministe constitue un deuxième facteur de changement. Né à la fin des années 60 et dans les années 70, il a conduit à la féminisation de la profession d'avocat et à une forme de revendication beaucoup plus agressive en faveur des droits des femmes à l'occasion du divorce. De 1960) à 1980, la proportion de femmes parmi les étudiants en droit est passée de 3,2 \% à 34,3\%. En 1985, elle était de $40 \%{ }^{13}$. De même, les femmes avocates constituaient $3 \%$ de la profession en 1960 et $12 \%$ en 1980. Par la suite, 
leur nombre a pratiquement double, puisquelles representaten $22 \%$ de la profession en $1990{ }^{14}$. Bien que beaucoup de e's fem mes qui sont entrees au barreau alient pris des postes dans les grandes études de droit des affaires ou soient devenues juristes dans des instances gouvernementales, il reste qu'une partic d'entre elles s'est trouvée engagée dans la pratique du droit de la famille.

Depuis les années 6(), les femmes avocates sont plus souvent en charge des affaires de divorce que ne le sont les hommes ${ }^{15}$. Certaines femmes sont tenues de traiter ce type de dossier en raison des stéréotypes sexués qui continuent de prévaloir dans les ćtudes où elles travaillent, tandis que d'autres choisissent de s'intéresser au divorce au raison de l'intérêt de ces affaires et du style d'interaction qu'elles impliquent avec les clients. Enfin, certaines avocates considerent que le droit du divorce constitue un champ privilégié de réalisation des idéaux féministes et s'y adonnent pour faire avancer les droits des femmes. Elles associent par conséquent la pratique privée habituelle à l'avocat du divorce avec un militantisme public plus large en faveur des droits civiques.

Étant donné l'accroissement rapide du nombre des femmes au barreau et la proportion assez large de celles qui pratiquent le droit du divorce, il est possible d'utiliser ce domaine pour aborder la question de savoir si le fait d'être homme ou femme fait une différence dans la pratique des avocats ${ }^{16}$. Les femmes apportent-elles dans le champ juridique et judiciaire des valeurs, des sentiments et des modes de comportement qui leur sont spécifiques? Ou bien, peut-on penser au contraire que la rigidité de l'apprentissage du droit et que le caractère uniforme des structures juridiques et de la pratique de l'avocat effacent toute différence de "genre" qui pourrait subsister entre les avocats? Ou bien encore, ne trouve-ton pas dans la profession d'avocat, hommes et femmes confondus, toute la diversité inhérente à n'importe quel ensemble humain? Nos entretiens avec les avocats du divorce font ressortir quelques différences importantes dans leur façon de pratiquer suivant qu'ils sont hommes ou femmes. Mais comment savoir si ces différences sont véritablement le reflet d'une orientation liée à leur sexe ou seulement la résultante de leur spécialisation et de la nature de leur clientèle?

Avocats et avocates ne se départagent pas seulement dans leur manière différente de pratiquer (gender roles): le degré de leur spécialisation en matière de divorce est également différent, ainsi que les catégories sociales auxquelles appartiennent leurs clients. Dans notre échantillon d'avocats, les deux tiers de ceux dont les dossiers sont constitués à plus de $75 \%$ par des divorces sont des femmes. À l'inverse, les femmes ne représentent que $7,5 \%$ des avocats ayant moins de $25 \%$ d'affaires de divorce dans leur pratique. En même temps, comme on l'a noté, les spécialistes du divorce ont des clients plus aisés. C'est ainsi que se fait jour, parmi
Droit et Sockite $3.3-1930$

14. Ibid., p. $284 ;$; E. EPSTEIN, Women in Law, 2" ed., Urbana, University of Illinois Press, 1993, p. 426.

15. J. WHITE, « Women in the Law", Michigan Law Review, vol. 65,1967, p. 1062-1063.

16. Dans un article antérieur, nous mettons en évidence ces différences dans l'orientation du rôle de l'avocat en fonction de son sexe et de sa spécialisation (R. MAINAN, L. MATHER et C. MCEWEN, "Gender and Specialization in the Practice of Divorce Law ", Maine Law Review, vol. 44, n 1, 1992 , p. 39-61). Parmi les travaux importants sur ces questions, citons : C. MENKEL-MEADOW, "Portia in a Different Voice : Speculations on a Women's Lawyering Process ", Berkeley Women's Law Journal, vol. 1 , 1985, p. $39-63$; C. MENKELMEADOW, « Exploring a Research Agenda of the Feminization of the Legal Profession: Theories of Gender and Social Change ", Law \& Social Inquin, vol. 14 , n 2, 1989, p. 289-319; C. EPSTEN, op. cit. ; R. JACK ot D. JACK, Moral Vision and Professional Decisions: The Changing Values of Women and Men lawyers, New York, Cambridge University Press, 1989 et J. PIIRCE, Gender Trials, Berkeley, Iniversity of California Press, 1995. 
L. Mather, R.J. Maiman,

C.A. McEwen

Avocats et divorce

aux États-Unis :

la transformation des

pratiques professionnelles les avocats, une représentation selon laquelle le rapport entre sexe et type de pratique est associé aux différences entre spécialistes et généralistes. Dans la mesure où il y a si peu de femmes parmi les généralistes, on en arrive à concevoir qu'il existe une modalité typiquement féminine de la pratique de l'avocat, qui est en fait celle des femmes spécialisées en matière de divorce.

Quelques femmes développent un style de pratique particulièrement agressif qui repose sur l'usage de procédures formelles davantage que sur les pourparlers entre confrères visant à échanger des renseignements au sujet des affaires. Elles sont d'ailleurs perçues comme telles par leurs confrères masculins. Ces femmes se montrent fières de la vigueur de leur engagement envers la cause de leurs client(e)s et elles ont parfois le sentiment qu'elles doivent " en faire beaucoup " pour être prises au sérieux par certains de leurs confrères hommes, plus anciens dans la profession. Par exemple, dans les deux États que nous avons étudiés, quelques femmes ayant une pratique individuelle d'avocate - toutes spécialisées en matière de divorce - indiquaient qu'elles étaient personnellement responsables de l'introduction de procédures d'interrogatoire dans ces affaires. Au lieu de faire par téléphone le travail d'échange d'informations avec le confrère au sujet des ressources du couple, elles ont poussé à l'usage de documents légaux faisant état des biens et des ressources du couple ainsi que d'autres informations. Une de ces avocates justifiait ce changement avec ironie, en arguant que bien peu d'avocats voudraient régler la faillite d'un de leurs clients en se fondant sur des assurances verbales et qu'elle ne voyait pas pourquoi il lui faudrait accepter ça dans un divorce.

"Lorsque j'ai commencé à pratiquer le droit de la famille, j'ai conservé beaucoup des techniques procédurales que j'avais utilisées dans toutes sortes d'autres contentieux. Je dois au client de ne pas accepter de croire que son conjoint a une retraite de 20000 dollars juste parce que quelqu'un me dit qu'il en est ainsi. Si cela implique des interrogatoires et des comparutions de témoins, eh bien, il y en aura... Je vois beaucoup de mauvais travail fait par des avocats qui prétendent faire "ami-ami", et moi, je ne marche pas là-dedans. ”

Une autre femme décrivait comment elle avait commencé à utiliser ces modalités formelles d'instruction dans le domaine du divorce au début des années 80 . Elle s'était alors heurtée à une hostilité très forte de ses pairs.

"Quand j'ai commencé à prendre des divorces et à faire des interrogatoires, $j$ 'ai eu de nombreux appels de confrères qui m'injuriaient au téléphone et qui me disaient : "C'est impensable." Pour eux, c'était comme si les interrogatoires ne faisaient pas partie de la pratique du divorce. " 
Ces femmes choquaient souvent leurs confreres, mais persistaient dans leur volonté de chianger les pratiques dans le sens d'une plus grande attention au détail et d'un plus grand formalisme. Les résistances face à ce nouveau style de travail des avocates du divorce ne surprennent pas: la perception très négative qu'en ont les avocats généralistes masculins non seulement témoigne d'un certain sexisme, mais emprunte aussi aux critiques qui visent les spécialistes.

"En schématisant un peu, je dirais que certains des avocats les plus agressifs auxquels j'ai eu affaire étaient des avocates. Je pense qu'elles font montre de peu d'intérêt pour essayer de résoudre les problèmes en travaillant sur les divergences et en cherchant un règlement amiable. "

Ces propos sont de ceux que tiennent les avocats généralistes hommes ayant des clientèles populaires ou des clientèles allant jusqu'à la classe moyenne. En se montrant, à contrecœur, admiratif à l'égard de telles femmes, l'un de ces généralistes souligne à quel point leur pratique tranche.

«Depuis une dizaine d'années s'est développé ici un groupe de femmes avocates qui se spécialisent dans le divorce. Elles sont vraimont agressives et très compétentes... Et elles sont terribles. Je veux dire, elles en veulent... Je déteste avoir affaire à elles, mais elles sont bonnes, vraiment très bonnes!»

Le même avocat continuait en expliquant que par " agressif » il voulait dire que les femmes spécialisées dans les affaires de divorce cherchaient à gagner tout ce qu'elles pouvaient obtenir. Il ajoutait que ces avocates "représentent habituellement les femmes et les défendent ».

Cette idée qui a cours parmi les avocats, selon laquelle les avocates représentent davantage les femmes dans le divorce, se trouve confirmée par les données empiriques. Parmi les professionnels que nous avons interrogés, les avocates représentaient la femme dans $71 \%$ de leurs dossiers de divorce, tandis que leurs confrères masculins représentaient la femme dans $53 \%$ des affaires. Cette proportion se retrouve quel que soit le degré de spécialisation, ce qui laisse penser que la variable sexe est plus forte que le mode de structuration du milieu professionnel. Nous avons obtenu le même résultat à partir de l'analyse des 7000 dossiers dépouillés dans les juridictions du divorce : les avocates représentent le plus souvent l'épouse dans le divorce, tandis que les hommes représentent le mari et la femme dans une proportion équivalente. Le fait que les avocates aient maintenant si souvent pour clientes les femmes dans les procédures de divorce - dont on sait qu'elles incluent des enjeux très importants en termes de genres, les droits des femmes s'opposant à ceux des hommes - constitue un changement consi- 
L. Mather, R.J. Maiman, C.A. McEwen

Avocats et divorce

aux États-Unis :

la transformation des

pratiques professionnelles dérable dans la pratique du droit du divorce depuis les années 506).

Pour les avocates, en particulier pour celles qui sont spécialistes du droit de la famille, le divorce, c'est très important, et le client aussi. Savoir que le divorce appauvrit les femmes et avoir pris conscience du prix très élevé que celles-ci paient sur le plan émotionnel au moment du divorce, cela galvanise ces avocates et les pousse à se battre très activement pour appuyer les revendications de leurs clientes. Ces avocates se différencient elles-mêmes des praticiens généralistes qui, pensent-elles, traitent les affaires de divorce avec peu de sérieux et ne représentent pas leurs client(e)s avec l'énergie qu'ils mettent dans d'autres types de dossiers.

"Les avocats hommes ne comprennent pas que je prenne ces questions de divorce avec le même sérieux qu'ils portent, disons, aux affaires immobilières ou aux contrats. Ils ont tendance à voir ça comme une position très têtue et très véhémente... Souvent, les avocats sont très désireux de parvenir à un accord qui paraitrait satisfaisant dans une affaire commerciale. Je vous mentirais si je vous disais que ce n'est pas un bon résultat, mais il faut bien voir que, dans les situations de divorce, un montant très faible peut faire une grande différence. Je pense sincèrement que cela fait une différence énorme dans la vie de ces gens. Les femmes prennent le travail en matière de divorce beaucoup plus sérieusement que ne le font les hommes. "

Un autre plan sur lequel la pratique des avocates diffère de celle des hommes : leur approche de la prise de décision. Alors que la plupart des avocats du divorce, comme d'ailleurs l'ensemble des professionnels du champ légal, mettent en exergue la très grande indépendance de jugement dont ils font preuve vis-à-vis de leur client, les avocates expriment certaines inquiétudes. Les avocats hommes, interrogent-elles, n'auraient-ils pas tendance à profiter de la domination qu'ils exercent sur leurs clientes pour promouvoir les solutions qu'ils préconisent, davantage que pour redonner du pouvoir aux femmes en situation de divorce?

"Je vois la différence d'approche dans le fait que les avocats ont tendance à faire la décision pour leur cliente; ils deviennent euxmêmes le principal décideur, au lieu de chercher à impliquer la cliente dans le processus de décision. Je connais beaucoup d'avocats qui prennent les décisions pour leur cliente et celle-ci perd tout pouvoir et tout contrôle - autrement dit, il se passe ici exactement la même chose que ce qui s'est passé pour elle pendant toute sa vie. "

Lorsqu'on considère la manière dont les avocats décrivent les objectifs qu'ils se fixent ainsi que leurs orientations professionnelles, on trouve donc une différence très forte entre spécialistes et non-spécialistes. Tandis que les premiers se voient répondre à l'ensemble des demandes du client (sur le plan émotionnel, économique ou légal), les seconds pensent devoir considérer d'une 
manière plus précise les attentes du client sur le plan juridique. Mais en y regardant de plus pres, et en se limitant aux specialistes, on constate encore une différence tres nette liée au sexe: les hommes sont deux fois plus nombreux que les femmes à définir leur principal rôle en termes de réponses aux besoins juridiques plutôt qu'en des termes plus larges renvoyant à l'ensemble des besoins du client ${ }^{17}$.

\section{Les changements du droit du divorce et le travail de l'avocat}

Les changements du droit du divorce sont la conséquence de l'accroissement du nombre des ruptures à partir des années 60 (époque où le divorce a été plus facilement admis dans la société), en même temps que de l'action du mouvement féministe qui a appelé à l'égalisation des droits et des responsabilités des hommes et des femmes au plan légal.

Sous l'ancien régime du divorce pour faute, les avocats devaient s'entendre avec leurs clients pour construire des torts, souvent fictifs, comme un adultère, de manière à satisfaire aux conditions légales très strictes en ce qui concerne la cause du divorce ${ }^{18}$. À partir de la libéralisation du droit du divorce à New York en 1966 et de l'introduction du "no-fault divorce " en Californie en 1969, on a assisté à un bouleversement complet du droit du divorce aux États-Unis ${ }^{19}$. Sous l'empire des nouvelles lois, les parties ont pu obtenir un divorce en faisant valoir qu'elles avaient des divergences inconciliables et que le lien conjugal était détruit d'une manière irréversible. Les autres États ont suivi en introduisant à leur tour des dispositions semblables et ils ont touché en outre à bien d'autres aspects du droit du divorce au cours des années 70, de manière à égaliser les situations des hommes et des femmes et à faire disparaître les privilèges accordés à un sexe sur l'autre : égalisation au plan de la répartition des biens, réduction de la dépendance induite par le versement de pensions alimentaires et changement de leurs modalités de fixation, recherche d'un meilleur équilibre entre père et mère dans l'attribution des droits parentaux ${ }^{20}$. Le travail législatif et les décisions judiciaires des années 80 ont remis en cause les dispositions traditionnelles fondées sur le sexe (préférence pour la femme en ce qui concerne la garde des enfants et paiement des pensions alimentaires dans le sens exclusif des hommes vers les femmes) pour aller dans le sens de la recherche de nouvelles règles caractérisées par leur neutralité au regard des sexes ("gender-neutral»).

Quel est l'impact de ces changements légaux pour les avocats du divorce ? La mise à l'écart de la notion de faute a fait disparaître les conflits portant sur la responsabilité dans la désunion. ì l'inverse, les changements juridiques relatifs à la division de la
17. Pour davantage de précisions sur ce point, on se reportera à R. MAIMAN, L. MATHER et C. MCEWEN, op. cit.

18. H. O' GORMAN, op cit., p. 20-25.

19. H. JACOB, Silent Revolution : The Transformation of Divorce Law in the United States, Chicago, University of Chicago Press, 1988.

20. E. MACOBY et R. MNOOKIN, Dividing the Child: Social and legal Dilemmas of Custody, Cambridge, Harvard Iniversity press, 1992. 
L. Mather, R.J. Maiman, C.A. McEwen Avocats et divorce aux États-Unis : la transformation des pratiques professionnelles

21. R. MNOOKIN et L. KORNHAUSER, "Bargaining in the Shadow of the Law : The Case of Divorce", Yale Law Journal, vol. 88, 1979, p. $950-997$.

22. A. SARAT et W. Felstiner, Divorce Lawyers and Their Clients : Power and Meaning in the Legal Process, New York, Oxford University Press, 1995, p. 112. propriété, aux pensions alimentaires et aux droits parentaux ont beaucoup accru les possibilités de conflit dans le règlement du divorce. Dès lors qu'aucune norme ne s'imposait plus pour l'attribution de la garde des enfants et pour la résolution des conflits sur les biens, et dès lors que l'idée se faisait jour que les hommes et les femmes ont les mêmes droits, les avocats du divorce ont été amenés à proposer les mêmes services à leurs clients, hommes ou femmes. Les affaires de divorce ont abouti à des solutions très variables, en fonction notamment des capacités de négociation des avocats. Dans leur travail classique sur la "négociation à l'ombre de la loi », Mnookin et Kornhauser ${ }^{21}$ ont d'ailleurs pris l'exemple du divorce pour mettre en évidence le caractère imprévisible du passage par le judiciaire et l'importance du rôle des avocats. Une recherche faite dans les années 80 en Californie et au Massachusetts a abouti exactement au même résultat $^{22}$.

Les avocats que nous avons interviewés au tout début des années 90 présentent cependant une image différente de l'activité en matière de divorce dans le Maine et le New Hampshire, avec moins de variabilité dans les décisions prises et une anticipation plus sûre des droits de chacun, du moins en ce qui concerne les questions financières. Comme le dit un avocat : "La plupart du temps, les gens savent ce qui va se passer. Le processus est très prévisible." Un autre indique: "La plupart des avocats plaidants expérimentés (trial lawyers) peuvent prévoir assez tôt quel sera le résultat d'un affaire de divorce avec une précision assez grande, voire avec certitude. " Les avocats expliquent d'ailleurs que les choses ont changé de ce point de vue : il y a une dizaine d'années, ce à quoi les divorçants pouvaient prétendre était plus flou. Depuis, les tribunaux ont clarifié leurs critères de décision pour la division des biens et pour la fixation du montant des pensions alimentaires. Dans les deux États, il existe une présomption selon laquelle les biens doivent être équitablement répartis dans un divorce non contesté. Les avocats considèrent que cela signifie un partage 50-50. Comme conséquence, ils ne recourent pas aux stratégies de combat qu'ils ont l'habitude d'adopter, par exemple en matière de réparation du préjudice personnel, et préfèrent une approche plus consensuelle de la négociation.

"Si vous avez affaire à un avocat qui connaît la loi, il n'y a rien à gagner à jouer ces jeux-là [adopter une stratégie offensive], parce que vous avez vite fait de savoir qui obtient quoi. Ce n'est pas pareil que dans les affaires de préjudice personnel : qui sait si une jambe perdue vaut un million de dollars ou un demi? Qui sait vraiment? Ici, cela n'a rien à voir. Vous devez faire avec la loi qui prévoit que cela va être du 50-50. »

En plus de clarifier les bases légales de la division des biens, les législateurs ont récemment introduit des règles (guidelines) 
pour la détermination du montant des pensions alimentaires pour les enfants. Le Congrès des États-Unis a poussé à l'adoption de telles règles en adoptant la loi fédérale de 1984 incitant les États à améliorer la prise en charge économique des enfants au moment du divorce. En réponse, l'État du Maine comme celui du New Hampshire ont introduit de nouvelles lois qui prévoyaient des normes mathématiques strictes pour le calcul des pensions alimentaires en fonction du revenu des parents et du nombre d'enfants. Près de $70 \%$ des avocats que nous avons interviewés considèrent que l'introduction de cette réglementation a rendu beaucoup plus facile leur travail en matière de divorce, en enlevant beaucoup de l'incertitude relative au résultat du procès et en réduisant par conséquent les zones de conflit entre les parties.

"Avant, c'était toujours à propos des pensions alimentaires pour les enfants qu'il fallait vraiment une séance de négociation jusqu'à ce qu'on se mette d'accord sur un montant. Souvent, on partait de très haut afin de se garder une marge pour pouvoir baisser. Avec les guidelines, ce n'est plus du tout pareil. »

Le résultat des procès n'est toutefois pas entièrement prévisible et c'est dans les zones d'incertitude qu'on voit se développer le travail juridique le plus intense. Par exemple, la clarification des règles sur la division du patrimoine n'empêche pas que subsiste la question de savoir quels biens doivent faire l'objet du partage. Les avocates féministes qui travaillent dans le champ du droit de la famille se sont appuyées sur ce point pour réclamer une définition plus large des biens matrimoniaux. Elles sont au courant des recherches qui démontrent que le divorce consensuel a des conséquences négatives pour la situation économique de la femme ${ }^{23}$ et elles pensent qu'en élargissant le " gâteau » les femmes pourront en obtenir une part plus grande. Leur activisme judiciaire a réussi et les plans de retraite (retirement funds) qui, jusqu'à présent, n'étaient pas pris en considération font maintenant partie des biens matrimoniaux. Conséquence : les avocats du divorce ont été amenés à s'intéresser de plus près aux aspects financiers et aux conséquences fiscales de la séparation. La complexité du règlement du divorce s'en est trouvée accrue, en particulier pour les clients ayant des revenus élevés.

Enfin, les questions relatives aux pensions alimentaires pour les enfants et aux droits parentaux, contrairement à ce qui se faisait traditionnellement, sont maintenant traitées selon des dispositions neutres en termes de sexe, ce qui revient à dire qu'elles offrent davantage de prise à la contestation légale. Il y a trente ou quarante ans, les mères se voyaient presque systématiquement accorder la garde des enfants. Aujourd'hui, les juges prennent au sérieux le critère du "meilleur intérêt de l'enfant " lorsqu'ils ont affaire à des cas contestés. La discrétion judiciaire reste grande et il n'est pas aisé de déterminer ce qu'est l'intérêt de l'enfant. De ce
23. L. WEITZMAN, The Divorce Revolution, New York, Free Press, 1985. 
L. Mather, R.J. Maiman,

C.A. McEwen

Avocats et divorce

aux États-Unis :

la transformation des

pratiques professionnelles fait, les tribunaux semblent accorder une grande importance à l'avis des spécialistes auxquels il est fait appel au cas par cas pour évaluer et protéger l'intérêt des enfants, les "guardians ad litem ".

Une dernière source importante de changement du droit du divorce réside dans les préoccupations de plus en plus vives qui s'expriment au sujet des difficultés ressenties par les enfants au moment du divorce. Ces préoccupations sont très présentes dans les propos des avocats au sujet de leur pratique. La plupart de ceux que nous avons interviewés indiquent que la présence d'en. fants dans la séparation les amène à adopter une approche plus consensuelle que celle qu'ils auraient autrement. Beaucoup insistent sur l'importance qu'ils attachent au fait de représenter au client les conséquences du divorce pour les enfants.

" Je leur dis: Vous avez deux enfants, un garçon et une fille, et vos enfants ont sept ou huit ou neuf ans... Je considérerais que j'ai fait du bon travail si, le jour où votre fille se marie, son père est là pour la marier et si, lorsque le pasteur demande qui conduit cette fille pour qu'elle soit mariée à cet homme, le père est là pour répondre : sa mère et moi ! Alors, je considérerais que j'ai fait du bon travail. Mais si j'ai le sentiment d'avoir semé les graines de l'amertume, c'est du mauvais travail. "

Les préoccupations des avocats à l'égard des enfants, pour authentiques et vécues qu'elles soient, restent généralement limitées au point de vue du client qu'ils représentent. Une poignée d'avocats du Maine ont cependant cherché à donner une autre ampleur à ce problème en constituant une organisation, formée principalement d'avocats et de travailleurs sociaux: "Ressources pour les familles divorcées» (RDF). Dans un premier temps, les membres de cette association ont échangé entre eux des informations au sujet des difficultés rencontrées par les enfants dans le divorce. Par la suite, ils ont offert des programmes visant à aider les divorçants à mieux prêter attention aux besoins de leurs enfants et ils ont cherché, sans succès, à obtenir du législateur de l'État du Maine que de tels programmes soient rendus obligatoires pour les couples divorçants ayant des enfants. Bien que le nombre des adhérents ait crû régulièrement et que l'association compte maintenant parmi ses membres des juges et des médiateurs professionnels, l'un de ses fondateurs nous a fait part de son amertume quant au fait de ne pas toucher les avocats, dont le nombre va sans cesse croissant, qui ne traitent qu'un petit nombre dc divorces chaque année. Pour la plupart de ces avocats, en effet, les questions relatives aux enfants restent un souci un peu vague, bien au-delà des préoccupations habituelles de leur travail quotidien, et elles affectent très marginalement leur pratique, voire même pas du tout.

Dans un certain sens, les efforts engagés par des associations comme "Ressources pour les familles divorcées", par la section de 
droit de la famille du barreau, ou encore par "The American Acad. emy of Matrimonial Lawyers " constituent un mouvement du droit de la famille qui pourrait bien ressembler à ce que l'on trouve dans certaines spécialisations médicales qui, "lorsqu'elles sont menacées, mettent au point une " formule de revitalisation" - autrement dit, trouvent un moyen d'incorporer de nouveaux aspects dans les missions traditionnelles de ce secteur ${ }^{24}$, ce qui améliore l'image du groupe professionnel. Dans ce sens, on a assisté, au cours des quarante dernières années, à un effort pour faire du travail des avocats en matière de divorce l'un des aspects importants de la pratique du droit de la famille, qui comporte par ailleurs d'autres dimensions, comme l'adoption ou la protection de l'enfance. De la même manière que certains avocats de Chicago cherchaient naguère à donner davantage de prestige à leur travail en matière de divorce en s'engageant dans le conseil conjugal et en s'identifiant ainsi à des professionnels de statut supérieur ${ }^{25}$, certains avocats du divorce choisissent aujourd'hui de se définir comme des "praticiens du droit de la famille» ( family law practitioners »). Un tel dispositif à fabriquer du prestige ne laisse pas de revêtir un caractère fortement symbolique. Alors que les avocats de Carlin voulaient qu'on identifie leur travail au souci de maintcnir la famille intacte, qu'ils faisaient valoir à travers des tentatives de réconciliation, les avocats de la famille aujourd'hui souhaitent qu'on se représente leur pratique à travers le symbole positif de la "famille ", plutôt qu'à travers celui plus négatif du " divorce ".

Le droit de la famille traite de la famille dans son unité originelle et dans sa forme recomposée, et pas seulement du divorce. En élargissant le champ pour considérer la famille, ce type de pratique peut aussi inclure une très large variété d'autres activités : adoptions, tutelles, ou mandats de "guardian ad litem». C'est ainsi que la profession d'avocat secrète, à travers l'activité de quelques spécialistes, une requalification permanente de la pratique du droit du divorce en pratique du droit de la famille, ce qui contribue à la construction du prestige professionnel de cette spécialité.

\section{Médiation et pratique du divorce}

Pour finir, les institutions qui traitent les affaires de divorce se sont modifiées dans les dernières décennies avec le projet de considérer le divorce comme une totalité au-delà de ses aspects juridiques. Maris et femmes ont été encouragés à exprimer leurs besoins sur le plan personnel et émotionnel et pas uniquement sur le plan légal, et ils ont été incités à prendre en charge eux-mêmes la définition de leur problème ainsi que sa résolution. Cette insistance sur la maîtrise du divorce par les parties en même temps que les préoccupations relatives aux effets du divorce conflictuel sur les enfants comme sur les parents ont contribué au développement

24. D. PAWLUCH, "Transitions in Pediatrics : A Segmental Analysis ", Social Problems, vol. $30, n^{\circ} 4,1983$, p. 460. 25. J. CaRIIN, op. cit., p. 97. 
L. Mather, R.J. Maiman, C.A. McEwen

Avocats et divorce

aux États-Unis :

la transformation des

pratiques professionnelles

\section{J. PEARSON, « Family}

Mediation ", in S. KeILITZ (ed.), National Symposium on CourtConnected Dispute Resolution

Research, Williamsburg, National Center for State Courts, 1994, p. 55.

27. Les données réunies par le National Center for State Courts pour sa base de données sur les dispositifs alternatifs de résolution des conflits (State ADR Database) indiquent qu'en 1991, $14 \%$ des programmes rapportaient que les avocats participaient habituellement aux séances de médiation. Voir C. MCEWEN, N. ROGERS et R. MAIMAN, « Bring in the Lawyers : Challenging the Dominant Approaches to Ensuring Fairness in Divorce Mediation", Minnesota Law Review, vol. 79, n` 6, 1995, p. 1317-1411.

28. Pour une analyse plus détaillée des conséquences de la médiation sur la pratique des avocats dans le Maine, voir C. MCEWEN, L. MATHER et R. MAIMAN, « Lawyers, Mediation, and the Management of Divorce Practice ", Law \& Society Review, vol. 28, n' 1, 1994, p. 149-186.

29. Dans d'autres systèmes judiciaires, des audiences préalables au procès peuvent avoir cette même finalité d'attirer l'attention sur la nécessité de parvenir à une solution - finalité que l'on trouve dans la médiation telle qu'elle est pratiquée dans cet Etat. du recours aux solutions alternatives de résolution des conflits. Les programmes de médiation en matière de divorce ont proliféré au cours des quinze dernières années. En considérant les juridictions chargées des affaires intrafamiliales, on constate qu'en 1994 une sur onze fonctionnait avec un dispositif de médiation obligatoire, tandis que dans un bon nombre d'autres la médiation était fortement encouragée ${ }^{26}$.

Les programmes de médiation en matière de divorce présentent une grande diversité eu égard aux types de situations et de problèmes qu'ils traitent, à la proportion de volontariat qu'ils incorporent, aux qualifications et à la responsabilité des médiateurs, etc. Ils diffèrent aussi suivant la manière dont ils associent les avocats à la médiation. De ce point de vue, les dispositifs qui prévoient la présence des avocats durant les séances de médiation restent l'exception ${ }^{27}$. Dans le Maine, la médiation est obligatoire dans tous les divorces contestés comprenant des enfants mineurs et les avocats ont l'habitude de participer avec leurs clients aux séances de médiation. La recherche que nous avons menée dans cet État montre bien à quel point le travail des avocats a été affecté par ce changement institutionnel ${ }^{28}$.

Telle qu'elle est pratiquée dans le Maine, la médiation en matière de divorce n'a pas détourné des affaires qui, autrement, auraient été prises en charge par des avocats et elle n'a pas interféré avec la capacité des avocats de représenter leur client d'une manière qu'ils jugent satisfaisante. Au contraire, les avocats considèrent que la médiation facilite généralement leur travail dans la mesure où elle procure des solutions et des ressources alternatives pour traiter les affaires. La médiation constitue un temps officiel dévolu au règlement du conflit, qui peut contribuer à faire prendre conscience beaucoup plus tôt aux parties de la nécessité de parvenir à un règlement ${ }^{29}$. Elle propose une alternative structurée au processus de discussion habituel entre avocats, plus flou et disjoint, dans lequel les positions extrêmes prises par les conseils ralentissent souvent la négociation. Comme l'indique un avocat :

"Les avocats ne se parlent jamais en face à face. C'est facile de jouer Tarzan au téléphone, vraiment. C'est plus difficile de faire ces conneries-là devant le client. Au téléphone, vous pouvez facilement dire de ma cliente qu'elle est une salope ou de mon client qu'il est un escroc, mais c'est vraiment différent d'avoir le culot de le faire quand ils sont de l'autre côté de la table. Beaucoup d'avocats sont capables de ces bêtises au téléphone, mais pas en face à face. "

Les avocats sont nombreux à dire aussi que la médiation les aide à cadrer les attentes des clients et à influencer leurs décisions. En particulier, lorsque la médiation donne l'occasion d'un " test de réalité " au sujet des revendications déraisonnables qu'exprime l'une des parties par rapport à l'issue du procès. 
"Le mieux, c'est que la médiation amène les clients à toucher du doigt la réalite dans certaines situations, quand je n'arrive pas à les ramener à la raison sur un point où ils restent dans des attentes exagérées, ou encore quand ils soupçonnent que je n'ai pas suffisamment à cœur leurs intérêts... Alors, vous dites au client: "Vous ne pouvez pas faire ça! " et il va en médiation et il s'aperçoit que ce n'est pas seulement mon idée. Alors je peux y revenir et lui dire: "Vous voyez, je vous l'avais dit". C'est comme si cela leur donnait une seconde opinion. "

La médiation change le travail des avocats avec leur client d'une autre façon encore, en créant une sorte de forum où peuvent être discutées les questions autres que juridiques. Un quart environ des avocats interviewés se montrait intéressé par l'opportunité qu'offre la médiation aux divorçants d'exprimer certains sentiments qui autrement n'auraient pas d'exutoire et pourraient empêcher la mise au point d'un accord.

"Vous allez obtenir plus en vous mettant autour d'une table et en offrant aux parties la possibilité de dire ce qu'elles ont à dire. Peut-être qu'émerge alors un problème qui n'a absolument rien à voir avec ce qu'il y a à décider. Et peut-être que vous pouvez faire sortir ce problème et faire qu'il ne reste pas en travers de la voie. Par exemple, la femme peut être furieuse uniquement parce que l'homme commence à sortir avec une autre femme. Peut-être pourrez-vous retirer cette épine, mettre ça sur la table. »

La plupart des avocats interviewés dans le Maine pensent que la médiation obligatoire a réduit l'affrontement auquel donne lieu habituellement le procès en divorce. On en trouve une confirmation modeste en comparant le nombre moyen des adversarial motions par affaire de divorce dans l'État du Maine et dans celui du New Hampshire, un État dans lequel n'existe pas de médiation auprès des tribunaux et très peu de médiation dans le secteur libéral. Dans le New Hampshire, la fréquence de ces requêtes est passée de 0,77 par dossier en 1980 à 1,06 en 1988, tandis que, dans le même temps, elle a diminué de 1,14 à 0,92 dans le Maine.

\section{Conclusion}

En définitive, le travail des avocats en matière de divorce a beaucoup changé aux États-Unis depuis quelques dizaines d'années. Alors que le nombre des divorces s'est stabilisé après des années d'accroissement très rapide, un grand nombre de ces affaires se présentent chaque année, de sorte que le divorce procure une clientèle très régulière aux avocats qui se spécialisent dans le champ de la famille. Le nombre croissant des couples aisés qui divorcent a d'ailleurs poussé une fraction des avocats à investir beaucoup de temps dans ce type de travail, avec des taux horaires élevés et par conséquent une pratique lucrative. Par ailleurs, un 
L. Mather, R.J. Maiman, C.A. McEwen Avocats et divorce aux États-Unis : la transformation des pratiques professionnelles nombre considérable d'autres avocats comptent aussi sur les affaires de divorce - celles qui concernent les membres des classes moyennes et populaires - pour constituer une part de leur activité de généraliste. Spécialistes et généralistes se différencient autant dans leur manière d'approcher la situation de leurs clients que dans les stratégies judiciaires qu'ils mettent en œuvre. En dernier lieu, une grande proportion de ces spécialistes sont des femmes, avec une clientèle constituée davantage de femmes que d'hommes, puisque les avocates représentent davantage les femmes dans le divorce.

La réforme des dispositions touchant à la division des biens et les nouvelles préoccupations concernant l'équité entre mari et femme ont conduit à recourir à des procédures formelles d'instruction des affaires qui font que le divorce des couples aisés ressemble aujourd'hui à la dissolution des relations entre partenaires dans le monde des affaires. En même temps, les réformes législatives allant dans le sens d'une plus grande équité et d'une meilleure prévisibilité du résultat des procès, en particulier en ce qui concerne la division du patrimoine et le montant des pensions alimentaires pour les enfants, ont réduit les zones de contestation juridique et simplifié le travail des avocats.

Enfin, le recours à la médiation a offert aux avocats un lieu de prise en charge des besoins de leurs clients sur le plan affectif, en même temps qu'un moyen pour canaliser leurs attentes et pour aider à la recherche de décisions consensuelles. La médiation semble aussi, au moins dans le Maine, avoir réduit le conflit entre les parties dans le divorce.

Beaucoup de ces changements ont contribué à "professionnaliser " la pratique du divorce et ont poussé à des efforts collectifs pour améliorer l'image et le prestige du travail des avocats dans ce champ. Cependant, les efforts ainsi développés par les spécialistes du divorce et du droit de la famille pour s'organiser ne font qu'accroître la distance qui sépare la perception qu'ils ont de leur pratique et celle qu'en a la masse des avocats qui traitent du divorce occasionnellement, parmi bien d'autres affaires. 